\title{
CLINICAL PROCEDURES
}

\section{Surgical hygiene}

\section{Refining the WHO Safe Surgery Saves Lives guidelines}

Alexander Levit (Meds 2017), Denise Darmawikarta (Meds 2016)
Faculty Reviewer: Dr Javeed Sukhera, MD (Department of Psychiatry, Office of Global Health)

\begin{abstract}
Overwhelming rates of postsurgical adverse outcomes have been recognized to be preventable. As a means of minimizing the incidence of iatrogenesis, in 2005 the World Health Organization (WHO) launched the Clean Care is Safer Care campaign, which focused on the importance of hand hygiene. Following its success, the WHO hoped to utilize similar strategies in their Safe Surgery Saves Lives campaign, which began in 2007. Despite active efforts to implement the associated 19-step surgical checklist, resource limitations restrict the full potential of such harm reduction strategies. Here, we discuss current evidence evaluating the effectiveness of the WHO's surgical checklist and address challenges with respect to its application in the real world.
\end{abstract}

\section{INTRODUCTION}

Iatrogenic injury, inadvertent harm or illness caused by medical therapy, remains a significant issue due to its impacts on patient outcomes and physician practice. To address the persistently high incidence of iatrogenesis, the World Health Organization (WHO) passed a resolution in 2002 that identified patient safety as a global priority. This prompted a series of internationally coordinated awareness campaigns. The first campaign began in 2005, was entitled Clean Care is Safer Care and emphasized proper hand hygiene and recommendations for improved sanitation practices. As of 2013, 15700 health care facilities worldwide have committed to the WHO guidelines for improving hand hygiene. ${ }^{1}$

The second WHO campaign, Safe Surgery Saves Lives, began in 2007 and aimed to reduce preventable causes of surgical complications. It is estimated that 234 million surgical operations are performed annually around the world, ${ }^{2}$ with $3 \%$ to $17 \%$ of procedures resulting in major complications and $0.4 \%$ to $0.8 \%$ in perioperative death. ${ }^{3}$ At least half of these adverse outcomes are thought to be preventable, quantified as approximately 468000 preventable deaths every year. ${ }^{3}$ While the statistics urge a widespread harm reduction intervention, the complexities of surgical error make it much more difficult to address than hand hygiene. Hand hygiene guidelines that were put forward in the Clean Care is Safer Care campaign were effective because they followed the principles of simplicity and wide applicability. In contrast, the crucial and resource-sensitive challenges of surgery that are specific to any one ward, hospital or country can be too unique for wider application. Despite these challenges, the most recent WHO guidelines optimistically state that these same principles will be maintained in the Safe Surgery Saves Lives campaign. ${ }^{4}$

\section{THE CHECKLIST}

The cornerstone of the Safe Surgery Saves Lives recommendations is a 19-step surgical safety checklist that includes items for review prior to induction of anaesthesia and skin incision and before the patient leaves the operating room. An accompanying manual guides the clinician through proper use of the checklist. Checklist items include more universal perioperative considerations, such as patient identification, consent and proper site of surgery, and more specific instructions, such as a prompt for two intravenous access points if more than $500 \mathrm{~mL}$ of blood loss is anticipated. Communication of procedural or patient-specific concerns is also encouraged between members of the surgical team (nurse, anaesthetist and surgeon).

A large pilot study appraised the benefit of the WHO checklist in 8 hospitals around the world. The rate of perioperative death from noncardiac surgery averaged $1.5 \%(n=3733)$ before intervention, and decreased significantly to $0.8 \%(n=3955)$ after checklist training and implementation. ${ }^{3}$ In the same population samples, inpatient complication rates also decreased significantly from $11.0 \%$ to $7.0 \%$. These statistics align well with the estimate that half of surgical injuries are preventable and suggest that the checklist can accomplish a meaningful reduction in these events. However, the study is plagued by several limitations. The researchers of the study pointed out that surgical team performance may have improved during use of the checklist as it could have heightened the teams' awareness that they were being observed. They also noted that patient complications were only observed up until discharge, suggesting that the incidence of postsurgical complications could have been underestimated.

It is important to note that the above study may lack generalizability. While 4 of 8 hospitals belonged to low- or middle-income countries, only one hospital was not a large tertiary hospital in an urban centre. This lack of diversity raises concerns regarding the applicability of the study results to a broader range of hospital settings, specifically smaller or rural facilities. Pulse oximetry and prophylactic antibiotics were identified in the study as requiring the commitment of significant resources, and were available at the low-income sites but used inconsistently prior to checklist intervention. The study made no comment on whether the increased use of these resources placed any strain on hospital supplies. Given the estimate that half of the world's population lives in a rural setting, ${ }^{8}$ it was also misrepresentative to recruit only one rural hospital. Though recruitment of hospitals from more truly diverse settings would have yielded more convincing data, herein could arise the problem of global variability. For instance, pulse oximetry was considered to be a superfluous checklist item for United Kingdom (UK) anesthetists as it is standard. ${ }^{5}$ Meanwhile, in developing countries, where preventable mortality attributed to anaesthesia can be as high as 1 in $150,{ }^{9}$ pulse 
oximetry may frequently not be available and a serious shortage of trained anaesthetists may be a more pressing issue altogether. ${ }^{10,11}$

Additionally, a smaller independent study on the implementation of the checklist suggested that certain surgical subspecialties will need to modify the checklist. For instance, orthopaedic surgery will benefit from checking for prophylaxis against deep vein thrombosis. ${ }^{5}$ The WHO guidelines advise additions and modifications of the checklist to fit local practice, but do not address the inherent problems created by a list that becomes too lengthy. Research suggests that when a list becomes too exhaustive, there is an increased likelihood for any checklist item to be overlooked. ${ }^{6}$ Furthermore, studies in aviation have pointed out that a poorly executed checklist can give a detrimentally false sense of security. ${ }^{7}$

While these limitations may be amenable to optimization through modifications of the checklist and its implementation, matters of resource limitation, as discussed previously, could pose even greater challenges to the checklist's effectiveness. One UK anaesthetist accurately captured this issue in a comment on the checklist: "It is a bit artificial sometimes. It's trying to be everything to every nation and to every standard of medicine." 5

\section{THE BEST STANDARDS CHECKLIST}

As it stands today, the current checklist and guidelines aim to be widely applicable by falling somewhere in between ideal and resource-limited surgical safety standards. This results in a dichotomous insufficiency. For affluent regions with developed medical infrastructures, the checklist may be too basic and the benefit of the checklist restricted. On the other hand, for poorly-funded facilities, where the training or resources indicated on the checklist may not be available, the checklist would be unusable. We suggest that it would be more appropriate to maintain an updated checklist that focuses on the best standards of patient safety given the best reasonably available surgical resources. This, in combination with knowledge exchange and capacity-building strategies, may best pave the way to safer surgeries. Such a position would not neglect the right to safe surgery for patients in low- and middle-income countries; rather, it would recognize the more pressing issues of surgical availability and accessibility where they are limited.

\section{WHERE AVAILABILITY \& ACCESSIBILITY ARE A GRAVER CONCERN}

Though it may be beyond the scope of the WHO patient safety program to supply surgical resources to developing countries, it is imprudent of the Safe Surgery Saves Lives campaign to neglect this more critical challenge to safe surgical care. Given the importance of minimizing iatrogenesis, the developing world would be better served by the distribution of information on the safety of adaptations made for resource-limited surgery instead of financially inappropriate recommendations. Surgical innovations discovered in low- and middle-income countries have been very cost-effective but due to safety concerns and lack of knowledge dissemination, these innovations may take a long time before they are globally appreciated and adopted. ${ }^{12}$ Many such innovations like the Bogotá bag, the use of a mosquito net mesh for inguinal hernia repair, and manual small incision cataract surgery have drastically improved global surgical care. ${ }^{12}$ It would also serve the additional benefit of helping to dispel the il- lusion that essential surgeries cannot be affordable or cost-effective in developing countries. ${ }^{13}$ Indeed, the Safe Surgery Saves Lives campaign has already endorsed the Lifebox, a more affordable yet still reliable pulse oximeter developed by a nonprofit organization. ${ }^{14}$

\section{CONCLUSION}

While hand hygiene is relatively a more globally achievable measure, global surgical safety involves more procedure-specific guidelines and is more resource-sensitive. Simplicity and wide applicability helped to make the Clean Care is Safer Care campaign a success, but Safe Surgery Saves Lives could be made more successful with a more appropriate and stratified approach. We propose the following:

1. Specific guidelines should be adapted for specific areas of surgery.

2. Up-to-date checklists should be maintained and based on the best standards of care, with fewer superfluous checklist items.

3. More parallel effort should be made to endorse and disseminate safety information on surgical innovations adapted for resource-limited facilities.

This approach would help to improve both the safety and the accessibility of surgery on a global scale. Though preventable patient harm is a global issue, improving the safety of surgery requires local measures.

\section{REFERENCES}

1. Allegranzi B, Gayet-Ageron A, Damani N, et al. Global implementation of WHO's multimodal strategy for improvement of hand hygiene: a quasi-experimental study. Lancet Infect Dis. 2013 Oct;13(10):843-51.

2. Weiser TG, Regenbogen SE, Thompson KD, et al. An estimation of the global volume of surgery: a modelling strategy based on available data. Lancet. 2008 Jul;372(9633):139-44.

3. Haynes AB, Weiser TG, Berry WR, et al. A surgical safety checklist to reduce morbidity in a global population. N Engl J Med. 2009 Jan;350(5):491-9.

4. World Health Organization. WHO Guidelines for Safe Surgery 2009: Safe Surgery Saves Lives [Internet]. Geneva: World Health Organization; 2009 [cited Mar 5 2014]. Available from: http://www.ncbi.nlm.nih.gov/books/NBK143243/.

5. Vats A, Vincent CA, Nagpal K, et al. Practical challenges of introducing WHO surgical checklist: UK pilot experience. BMJ. 2010 Jan;340:133-5.

6. Swain AD, Guttman HE. Handbook of human reliability analysis with emphasis on nuclear power plant applications. Washington, DC: NRC; 1983.

7. Degani A, Wiener El. Cockpit checklists: concepts, design and use. Hum Factors. 1993 Jun;35(2):345-59.

8. Verbeek, J. Global monitoring report 2013: rural-urban dynamics and the millennium development goals [Internet]. Washington, DC: The World Bank; 2013 [cited Mar 5 2014]. Available from: http://go.worldbank.org/TLCNVQIJE0.

9. Ouro-Bang’na Maman AF, Tomta K, Ahouangbévi S, et al. Deaths associated with anaesthesia in Togo, West Africa. Trop Doct. 2005 Oct;35(4):220-2.

10. Hodges SC, Mijumbi C, Okello M, et al. Anaethesia services in developing countries: defining the problems. Anaesthesia. 2007 Jan;62(1):4-11.

11. Cherian MN, Merry AF, Wilson IH. The World Health Organization and anaesthesia. Anaesthesia. 2007 Dec;62 Suppl 1:65-6.

12. Cotton M, Henry JA, Hasek L. Value innovation: an important aspect of global surgical care. 2014 Jan;10(1):1

13. Disease Control Priorities Project. Promoting essential surgery in low-income countries: a hidden cost-effective treasure [Internet]. Washington, DC: Disease Control Priorities Project; 2008 [cited Mar 8 2014]. Available from: http://www.dcp2.org/file/158/dcpp-surgery.pdf

14. Dubowitz G, Breyer K, Lipnick M, et al. Accuracy of the Lifebox pulse oximeter during hypoxia in healthy volunteers. Anaesthesia. 2013 Dec;68(12):1220-3. 